\title{
Simultaneous manipulation of lignin structure and secondary cell wall formation in transgenic poplar
}

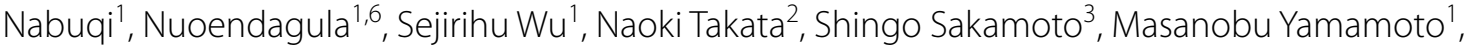 \\ Mikiko Uesugi ${ }^{1}$, Annabelle Déjardin ${ }^{4}$, Gilles Pilate ${ }^{4}$, Toru Taniguchi ${ }^{2,7}$, Nobutaka Mitsuda $^{3}$ and Shinya Kajita ${ }^{1,5^{*}}$ (D)
}

\begin{abstract}
Increasing the wood density to a preferred range contributes to upgrading the value of wood as raw material. Lignin manipulation can also improve wood quality. This study attempted to accelerate secondary cell wall formation in transgenic poplar with an altered lignin structure. To achieve this, OsSWN1, a rice master switch for secondary cell wall formation in fiber cells, was overexpressed in poplar plants in which monolignol biosynthesis was suppressed by the expression of an RNA-interference construct targeted to the gene for cinnamyl alcohol dehydrogenase 1. The generated transgenic poplars successfully overexpressed the chimeric OsSWN1 construct and monolignol biosynthesis remained altered in these plants. Secondary cell wall thickness was increased in the transgenic plants and their wood density was higher compared to the background line. No difference in lignin content was observed, except in one transgenic line. High saccharification characteristics observed in the background line were preserved in the transgenic line with lower OsSWN1 overexpression, but higher OsSWN1 overexpression had a slight negative impact on enzyme saccharification. Our data suggested that fine-tuning of accelerated deposition of the secondary cell wall combined with alteration of monolignol biosynthesis should improve the lignocellulose quality for conventional and future biorefinery uses.
\end{abstract}

Keywords: Genetic engineering, Poplar, Transcription factor, Wood density, Xylem

\section{Introduction}

Lignin is a major chemical component of cell walls in terrestrial plant biomasses. This phenolic polymer is mainly built from three monolignols, $p$-coumaryl, coniferyl, and sinapyl alcohols, which are biosynthesized from phenylalanine or tyrosine via the cinnamate/monolignol pathway $[1,2]$. In the last step of monolignol biosynthesis in the cytosol, $p$-hydroxycinnamaldehydes are reduced by cinnamyl alcohol dehydrogenase (CAD) to form monolignols [3]. After monolignols are transported to the cell wall, they are radicalized by phenoloxidases such as

\footnotetext{
${ }^{*}$ Correspondence: kajita@cc.tuat.ac.jp

${ }^{1}$ Graduate School of Bio-Applications and Systems Engineering, Tokyo University of Agriculture and Technology, Koganei, Tokyo 184-8588, Japan Full list of author information is available at the end of the article
}

laccase and peroxidase, and formed $p$-hydroxyphenyl $(\mathrm{H})$, guaiacyl $(\mathrm{G})$, and syringyl (S) units in the lignin polymer [2]. In addition to monolignols, $p$-hydroxycinnamaldehydes and other phenolics are incorporated into lignin as minor units [4].

Lignin gives cell walls their physical strength to support upward plant growth, facilitates water transport in plants, prevents microbial attacks, and protects plants from UV irradiation. Although lignin exhibits various essential roles for plant growth and development, it is highly resistant to chemical and biochemical pretreatment for the efficient utilization of the plant cell wall as lignocellulosic biomass [5]. Even for partial removal of lignin from the lignocellulosic biomass, especially woody biomass, material processing generally requires a substantial amount of energy and chemical consumption.
Springer Open (c) The Author(s) 2020. This article is licensed under a Creative Commons Attribution 4.0 International License, which permits use, sharing, adaptation, distribution and reproduction in any medium or format, as long as you give appropriate credit to the original author(s) and the source, provide a link to the Creative Commons licence, and indicate if changes were made. The images or other third party material in this article are included in the article's Creative Commons licence, unless indicated otherwise in a credit line to the material. If material is not included in the article's Creative Commons licence and your intended use is not permitted by statutory regulation or exceeds the permitted use, you will need to obtain permission directly from the copyright holder. To view a copy of this licence, visit http://creativeco mmons.org/licenses/by/4.0/. 
Decreasing the lignin content and changing the lignin structure in plant cell walls by genetic engineering via the manipulation of lignin biosynthesis genes can reduce lignin resistance in pretreatment processes [5]. Extensive studies have been performed in this area using Populus as a model tree species [6]. For example, suppression of p-coumaroyl-CoA $3^{\prime}$-hydroxylase and cinnamoylCoA oxidoreductase in transgenic poplars induce lignin reduction and/or structural changes $[7,8]$. These modifications result in improved enzyme saccharification efficiency in the wood tissue of transgenic plants.

CAD downregulation via expression of antisense and sense RNA constructs also induces structural modification of lignin $[9,10]$. In addition, CAD suppression by a hairpin RNA construct and a nonsense mutation of CAD lead to reduced lignin content with an altered structure [11-13]. CAD reductions in woody species result in the incorporation of substantial amounts of $p$-hydroxycinnamaldehyde units, especially sinapaldehyde units, into lignin $[11,12,14]$. CAD suppression also results in an increase in the number of free phenolic groups in the lignin, which enhance the reactivity of lignin under alkaline conditions. This improves lignin degradation efficiency under alkaline conditions, and hence, improves saccharification efficiency after alkaline pretreatment [10, $11,15]$. Although lignin modification by genetic engineering targeting lignin biosynthetic genes can occasionally lead to abnormal plant growth and impose a yield penalty on plant biomass, no severe negative impact on biomass yield has been reported in woody plants showing CAD downregulation under greenhouse cultivation and in field trials $[10,11]$.

In addition to improved lignin extractability, increasing wood density within a preferred range contributes, at least in part, to upgrading wood value as a raw material for biorefinery usage. Greater wood density means higher biomass production per unit of land and a larger amount of the biomass-derived product from a reactor per unit of time if the plant growth and product yield are independent of the density.

One of the options for improving wood density by genetic engineering is overexpressing the transcriptional regulator of secondary cell wall (SCW) biosynthesis. Sakamoto et al. [16] showed that expression of the rice secondary wall NAC (No apical meristem, Arabidopsis transcription activation factor, and Cup-shaped cotyledon) domain protein 1 (OsSWN1) in transgenic poplars resulted in increased cell wall thickness in fiber cells and increased wood density. This protein is the ortholog of the master regulator of SCW formation in Arabidopsis, NAC secondary wall thickening promoting factor $3 /$ SCW associated NAC domain protein 1 (NST3/SND1) [17-21]. The growth of transgenic poplars expressing
OsSWN1 under the control of the NST3 promoter is indistinguishable from that of wild-type plants under soil cultivation [16]. OsSWN1 overexpression in poplars induces structural modification of lignin but no significant change in lignin content has been observed.

In the present study, we evaluated the synergetic effect of suppressed lignin biosynthesis and enhanced SCW deposition in poplar with a simultaneous downregulation of CAD1 and overexpression of OsSWN1.

\section{Materials and methods Poplar transformation}

A chimeric OsSWN1 construct under the control of the Arabidopsis NST3 promoter [16] was introduced into hpCAD19 transgenic poplar line (Populus tremula $\mathrm{x}$ Populus alba clone INRA 717-1B4) [11] in which CAD1 activity was suppressed by the cauliflower mosaic virus $35 \mathrm{~S}$ promoter-driven hairpin RNAi strategy. Tissue-preferential expression of the Arabidopsis NST3 promoter in secondary xylem and phloem fibers in stems, in addition to ray parenchyma and vessel elements, were visualized by a histochemical promoter-reporter assay of the transgenic poplar plants [22]. Conventional genetic transformation via an Agrobacterium-mediated procedure was performed following the method described by Leplé et al. [23]. Hygromycin $(10 \mathrm{mg} / \mathrm{L})$ was added to the culture medium for the selection of transformed cells and subsequent regenerated plants. The resultant transgenic lines were coppiced by cutting in the medium with hygromycin and cultivated in soil for further use. The cultivation was performed in a culture room at $25^{\circ} \mathrm{C}$ under long-day (16-h light:8-h dark) conditions.

\section{Semiquantitative and quantitative reverse transcription-polymerase chain reaction}

Stem tissues were collected from the 11th and 12th internodes of 4-month-old plants and immediately frozen in liquid nitrogen. Isolation of total RNA was performed using TRIzol (Thermo Fisher Scientific K.K., Tokyo). After DNase treatment of the resultant total RNA, 1st strand cDNA was synthesized using a Transcriptor First Strand cDNA Synthesis Kit (Roche Diagnostics K.K., Tokyo) for semiquantitative RT-PCR. DNA derived from CAD1 transcripts was amplified using GoTaq DNA Polymerase (Promega K.K., Tokyo). The ubiquitin transcript was also amplified as a positive control. PCR was performed in a cycling condition as follows: 3 min at $95{ }^{\circ} \mathrm{C}$ followed by 35 cycles of $30 \mathrm{~s}$ at $95{ }^{\circ} \mathrm{C}, 30 \mathrm{~s}$ at $55^{\circ} \mathrm{C}$ and $1 \mathrm{~min}$ at $72{ }^{\circ} \mathrm{C}$ with a final step at $72{ }^{\circ} \mathrm{C}$ for $5 \mathrm{~min}$. Reaction mixtures with the amplified products (10 and $5 \mu \mathrm{l}$ for $C A D 1$ and ubiquitin, respectively) were separated by $1 \%$ agarose gel electrophoresis. Quantitative RT-PCR was performed as described 
by Takata and Taniguchi [24]. First-strand cDNA was synthesized using a High Capacity RNA-to-cDNA Kit (Thermo Fisher Scientific). Real-time PCR was carried out using a StepOnePlus Real-Time PCR System with PowerUp SYBR Green PCR Master Mix (Thermo Fisher Scientific). Gene expression levels were normalized to those of $18 \mathrm{~S}$ ribosomal RNA. RNA samples were assayed in triplicate with three biological replicates. The gene-specific primers for PCR are listed in Additional file 1: Table S1.

\section{Histochemical analysis}

Stem segments were sampled from the 5th and 6th internodes of each transgenic plant. Tissue sections $(10-\mu \mathrm{m}$ thick) were cut using a microtome (model MTH-1, Nippon Medical \& Chemical Instruments Co., Ltd., Osaka). To measure cell wall thickness by image analysis, the cross sections were stained with $1 \%$ safranin solution and images were obtained using a microscope (model BZ-X810, Keyence Corporation, Osaka). Cell wall thickness of over 400 xylem cells excluding vessel elements for each transgenic line were analyzed semi-automatically using binarized images obtained from safranin-stained sections using the Local Thickness plugin of ImageJ 2.0.0 $[25,26]$. Lignin autofluorescence of an unstained thin section was monitored using a microscope equipped with a DAPI filter set (type OP-87762, Keyence Corporation; excitation filter, $340-380 \mathrm{~nm}$; dichroic mirror, $400 \mathrm{~nm}$; emission filter, 435-485 $\mathrm{nm}$ long pass).

\section{Lignin content and composition}

Lignin characterization was performed with stem (4 months old) after bark peeling. After the air drying for 1 month at room temperature, a top part (approximately $3 \mathrm{~cm}$ in length) of the dried stem wood was removed for measurement of density. Remaining part of the wood was pulverized with a Multi-Beads Shocker (MB1200C, Yasui Kikai Corporation, Osaka) until the wood powder passed through a $150-\mu \mathrm{m}$ screen. Cell wall residue (CWR) was prepared from the powder by sequential extraction using $50 \mathrm{mM} \mathrm{NaCl}$ solution, hot water, methanol, $99.5 \%$ ethanol, a mixture of ethanol and toluene $(1: 2, \mathrm{v} / \mathrm{v})$, and acetone. Lignin content in CWR (ca. $5 \mathrm{mg}$ ) was measured using an acetyl bromide protocol as described previously [25]. Monomeric composition of lignin in CWR was analyzed by thioacidolysis. Conventional thioacidolysis products were quantified by gas chromatography equipped with a flame-ionization detector after trimethylsilylation $[14,25,27]$. For lignin content and composition analyses, three biological replicates were analyzed for each plant line.

\section{Wood density}

Density of the air-dried wood pieces described above was calculated from volume and mass of each piece. Volume of the wood piece was measured on Archimedes' principle in a beaker filled with Milli-Q water on a conventional laboratory balance. Density of gently packed CWR was measured using a PCR tube ( $0.2 \mathrm{~mL}$ volume). Masses of same volume of Milli-Q water and packed CWRs in the tube were measured on the balance and then used to calculate the density by dividing the mass by the volume. Preparation of CWR was described above.

\section{Enzyme saccharification}

Saccharification efficiency was monitored after diluted alkali pretreatment. CWR (ca. $10 \mathrm{mg}$ ) was mixed with $300 \mu \mathrm{L}$ of $100 \mathrm{mM} \mathrm{NaOH}$ solution and heated at $120{ }^{\circ} \mathrm{C}$ for $1 \mathrm{~h}$. After cooling to room temperature, the solution was neutralized with diluted hydrochloric acid. Saccharification was then carried out in $50 \mathrm{mM}$ citrate buffer (pH 4.8) with Celluclast (Sigma-Aldrich C2730) and Cellobiase (Sigma-Aldrich C6105) at $50{ }^{\circ} \mathrm{C}$ for $14 \mathrm{~h}$. Sugars released from the samples were quantified by 3, 5-dinitrosalicylic acid assay using glucose as a standard [28]. Saccharification efficiency was calculated based on the amounts of released sugar and CWR without pretreatment. Three biological replicates were analyzed for each plant line.

\section{Results and discussion}

Transgenic poplar generation and OsSWN1 expression

The tissue-preferential pattern of NST3 expression met our objective, i.e., SCW formation was promoted in poplar stem xylem by the chimeric overexpression of OsSWN1 [22]. After the genetic transformation of the hpCAD19 line via an Agrobacterium-mediated transformation procedure, we obtained over 10 independent transgenic lines with the chimeric OsSWN1 construct. We selected four independent OsSWN1-expressing lines (lines $\# B, \# C$, \#E, and \#G) for further analysis based on their growth stability during successive propagation by in vitro cuttings in the culture medium. Although they could be maintained by sequential in vitro propagation for over a year, cuttings from lines \#C and \#G were relatively difficult to root compared to the wild-type and other transgenic lines (data not shown).

Figure 1A shows each transgenic line placed in two rows in a culture room after soil cultivation for 4 months. Although the height of line \#B $(60.5 \pm 13.7 \mathrm{~cm})$ was slightly lower than that of wildtype plants $(79.3 \pm 3.9 \mathrm{~cm})$, no statistical differences were detected at $P<0.01$ level among the wild-type and hpCAD-19 $(85.5 \pm 7.8 \mathrm{~cm})$ lines, and the plant lines \#B 


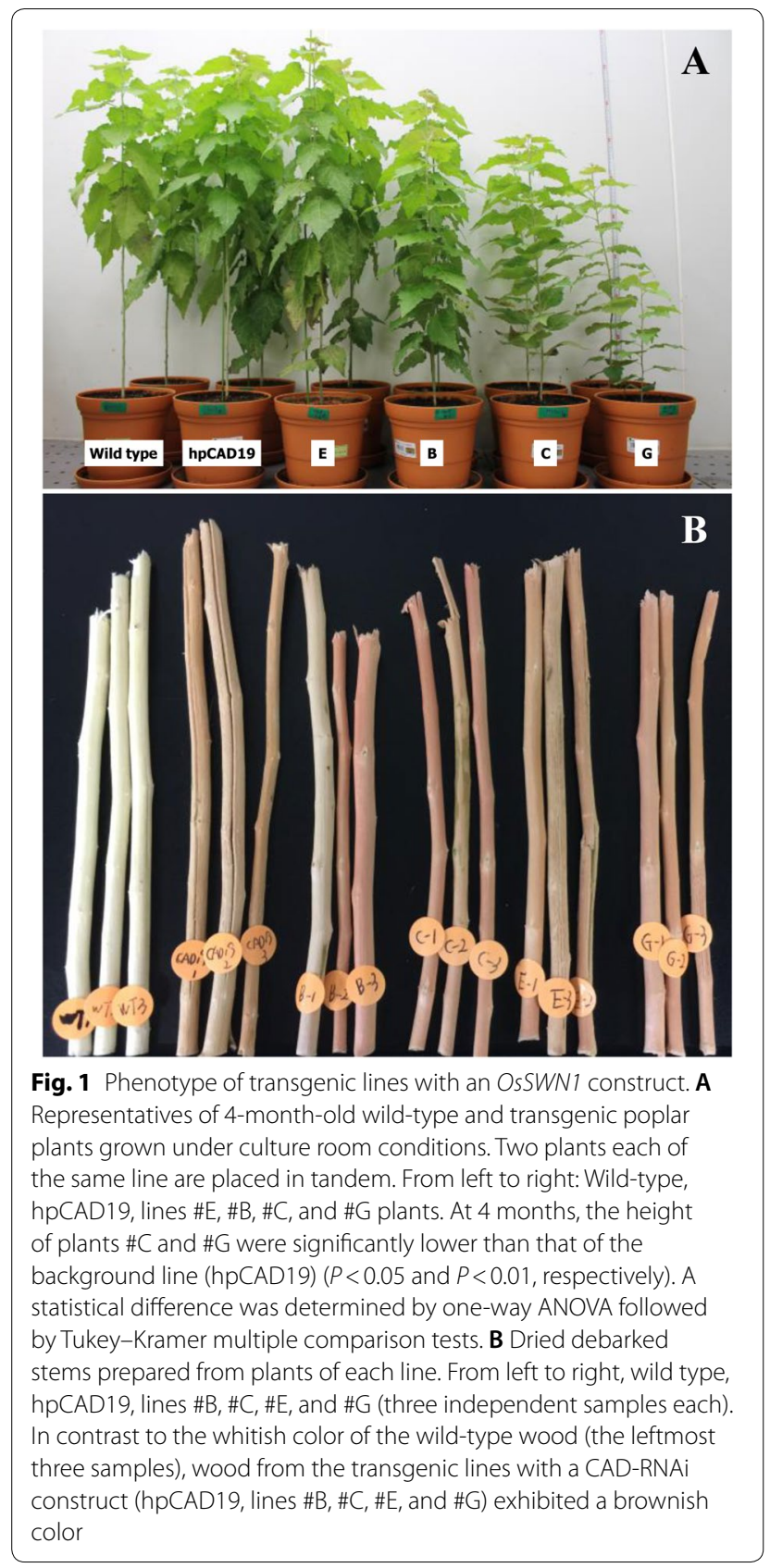

and \#E $(76.7 \pm 3.8 \mathrm{~cm})$. In contrast, growth retardation was observed in lines \#C $(54.0 \pm 7.3 \mathrm{~cm})$ and \#G $(33.5 \pm 17.7 \mathrm{~cm})$. This might be due, in part at least, to the introduction of the chimeric OsSWN1 construct into the plants.

To validate chimeric OsSWN1 expression, semiquantitative RT-PCR was performed using total RNA isolated from each plant. Expression was detected in the four OsSWN1 lines, but the OsSWN1 level in line \#E was apparently lower than that of the other OsSWN1 lines

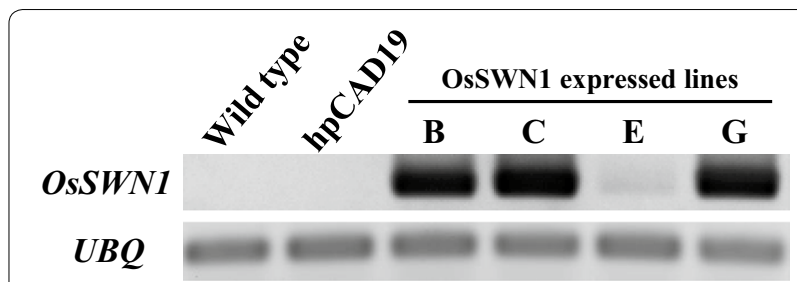

Fig. 2 Detection of transcripts from chimeric OsSWN1 by reverse transcription and subsequent polymerase chain reaction (RT-PCR). A primer pair that can amplify a part of the OsSWN1-coding region was used in this analysis. Transcripts from the ubiquitin gene were also analyzed as a control. Nucleotide sequences of the primers are indicated in Additional file 1: Table S1

(Fig. 2). These results indicated that OsSWN1 expression level varied among the different plant lines though difference in the expression level among the lines $\# \mathrm{~B}, \# \mathrm{C}$, and \#G was unclear in our present data. No amplified DNA derived from the transgene was observed in the wild-type and hPCAD19 plants.

\section{Endogenous CAD1 expression}

As reported previously, heterologous OsSWN1 expression can induce $C A D(C A D-D)$ expression in transgenic Arabidopsis [16] and CAD1 expression in transgenic poplar [25]. These results suggest that simultaneous expression of chimeric OsSWN1 and CAD1-RNAi transgenes is expected to result in a conflicting response on endogenous $C A D 1$ expression in the present lines. Thus, we checked whether sufficient CAD1 suppression could still be achieved in the four transgenic OsSWN1 lines. As indicated in Additional file 1: Figure S1, CAD1 expression remained depressed in all four OsSWN1 lines and no significant differences were detected compared to the hpCAD19 line, although the suppression levels varied slightly among the tested plants.

Unlike wild-type poplar, the CAD-deficient hpCAD19 line exhibited red coloration in its xylem tissue that turned to brown after drying (Fig. 1B). This coloration is a known indicator of $C A D$ deficiency in various plant species [14]. As indicated in Fig. 1B, the four OsSWN1 lines also showed brown xylem tissue coloration, indicating that endogenous $C A D 1$ expression was still efficiently suppressed in the transgenic poplars. This supports the findings of the real-time PCR analysis shown in Additional file 1: Figure S1.

\section{Histochemical analysis of stem tissues}

For further characterization of the effect of chimeric OsSWN1 expression on accelerating SCW formation, we analyzed lignin deposition and cell wall thickness in stems using thin sections of stem tissues. At lower 
magnification $(10 \times$ for wild type and $20 x$ for other lines) under bright field observation, no apparent histochemical differences were observed between the wild-type, hpCAD19, and OsSWN1-expressing lines (Fig. 3A-F). In contrast, microscopic observation of autofluorescence from plant tissues differed among the tested lines. No autofluorescence occurred in the pith parenchyma tissues of the wild-type (Fig. 3G), hpCAD19 (Fig. 3H), and line \#E (Fig. 3K) plants. In contrast, strong autofluorescence was detected in the three OsSWN1 lines $(\# \mathrm{~B}$, \#C, and \#G; Fig. 3I, J, L) in which the OsSWN1 expression was apparently higher than the line \#E, indicating that

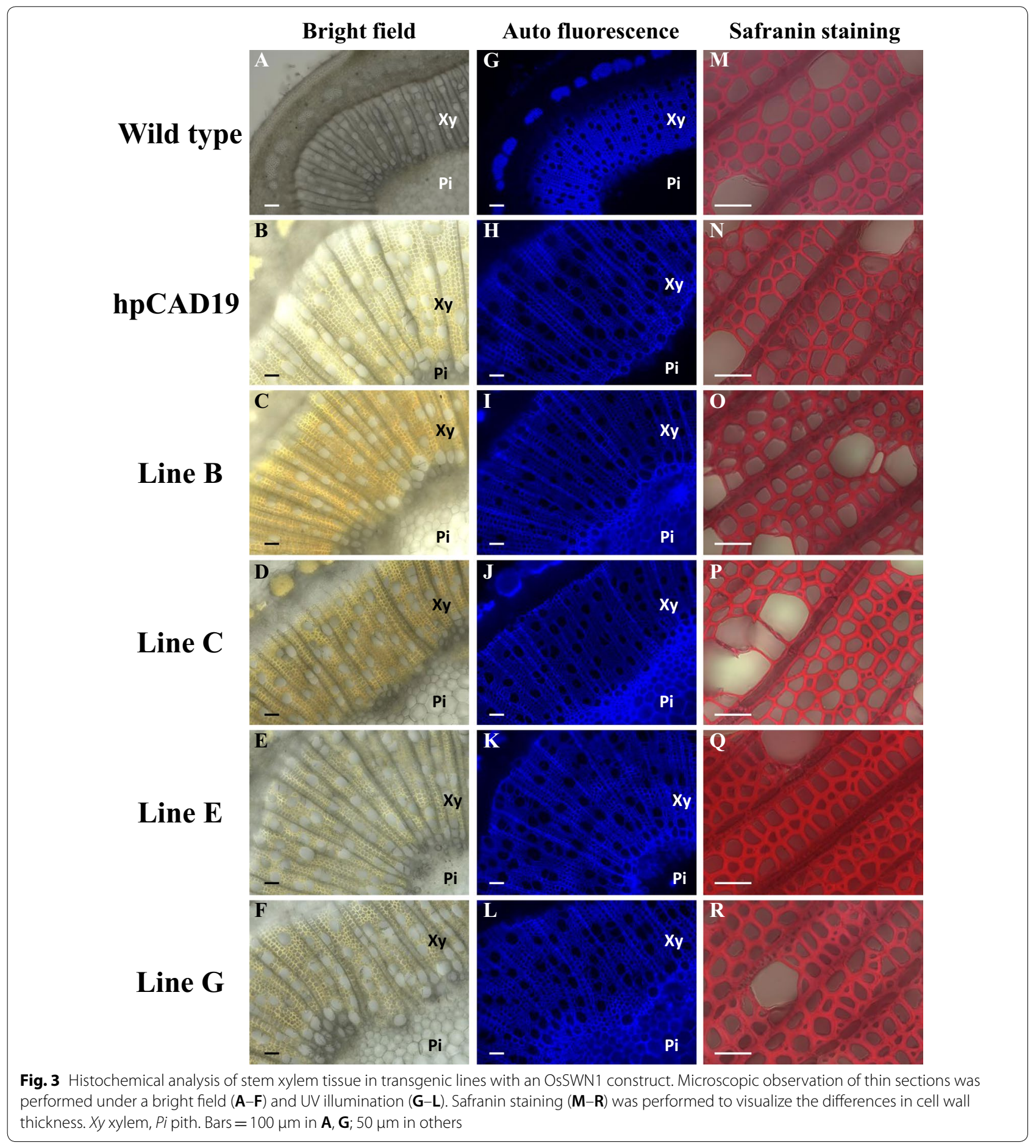


lignification was accelerated in the pith tissue, where less lignification generally occurs [29]. Accelerated pith lignification was previously reported in OsSWN1-expressing poplars generated in a wild-type background [16].

Under microscopic observation of safranin-stained sections at high magnification, cell wall thickness of xylem tissue in hpCAD19 plants seemed to be thinner than that of the wild-type plants, though the differences were not remarkable (Fig. $3 \mathrm{M}, \mathrm{N}$ ). In contrast, the cell wall thickness in lines \#B and \#G (Fig. 3O, R) were higher than those of hpCAD19 and wild-type plants. These results suggested that OsSWN1 expression induced enhanced cell wall formation in transgenic plants.

\section{Quantitative characterization of accelerated cell wall formation by imaging analysis}

To confirm the enhanced cell wall thickness in OsSWN1 lines, an image analysis of wood fiber cells was performed based on microscopic imaging of safranin-stained thin sections as described in previous studies [16, 25]. Analysis showed that the average cell wall thickness of fibers in the hpCAD19 line $(1.63 \pm 0.25 \mu \mathrm{m}, N=1405)$ was slightly but significantly lower than that of the wild-type line $(1.81 \pm 0.27 \mu \mathrm{m}, N=934)$ (Fig. 4). This result suggested that lignin modification by $C A D 1$ suppression accompanied reduced cell wall thickness in the fibers. In contrast, the cell wall thickness of the OsSWN1-expressing lines was significantly higher $(3.7 \%$ higher in \#E, $17 \%$ higher in $\# \mathrm{C}$, and $29 \%$ higher in $\# \mathrm{~B}$ and $\# \mathrm{G}$ ) than that of the

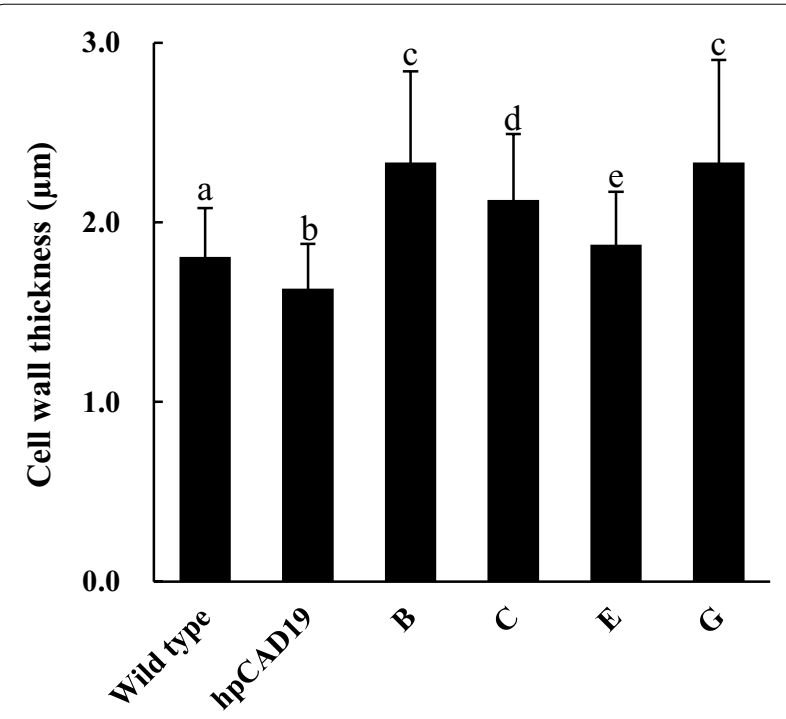

Fig. 4 Cell wall thickness of xylem cells measured by imaging analysis. Different letters indicate statistically significant differences between the various lines at $P<0.01$. A statistical difference was determined by one-way ANOVA followed by Tukey-Kramer multiple comparison tests. Error bars indicate the standard deviation wild-type line (Fig. 4). Our data indicated that OsSWN1 expression promoted cell wall thickening even under $C A D 1$ suppression and restored the reduced thickness observed in the hPCAD19 line.

\section{Moisture content in fresh wood}

Generally, enhanced wall thickness is expected to induce increased wood density. Since the moisture content of fresh wood has a roughly negative correlation with its density, we calculated the moisture content ([fresh weight - dry weight]/fresh weight) of debarked wood prepared from the stems of 4-month-old plants immediately after harvest and subsequent air-drying for 1 month under room conditions. Although wood moisture could not be completely removed by this procedure, moisture content of the whole stem samples could be estimated. As shown in Additional file 1: Figure S2, the moisture content of the wild type and hpCAD19 line were on the same level. In contrast, those in lines \#B and \#C were significantly reduced by $14 \%$ and $15 \%$, respectively, compared to the wild type. Compared to hpCAD19, the moisture content in lines \#B, \#C, and \#G were also significantly reduced by $15 \%, 16 \%$, and $7 \%$, respectively. In contrast, the moisture content in line \# $\mathrm{E}$ (in which the OsSWN1 expression was relatively low) was comparable to those of the wild type and the hpCAD19 line. Collectively, our results suggested that chimeric OsSWN1 expression led to decreased fresh moisture content in the debarked stem. The decrease might be due to decrease in intracellular space occurring along with accelerated thickening of SCW in xylem cells as previously reported in other transgenic poplars harboring OsSWN1 [25]. The enhanced SCW deposition also led to increase in wood density as discussed in the next section.

\section{Stem wood density}

The wood density of the top 3-cm part of harvested transgenic stems was determined based on Archimedes' principle (black bars in Fig. 5). The averaged values of three biological samples from lines \#B and \#C were 19\% and $24 \%$ higher than that of the wild-type plants, respectively. In contrast, the wood density of hpCAD19 plants was $15 \%$ lower than that of the wild-type plants. Although reduced wood density in hpCAD19 plants has not been previously reported, wood density in hpCAD24 line with the same CAD1-RNAi construct was significantly lower than that of its wild-type counterparts [30]. In contrast to the higher level of cell wall thickness enhancement detected in line \#G (Fig. 4), no difference in wood density was observed compared to that of the wild-type plant. This may partially be due to the differences in stem parts used in these analyses and the growth retardation of line \#G. 


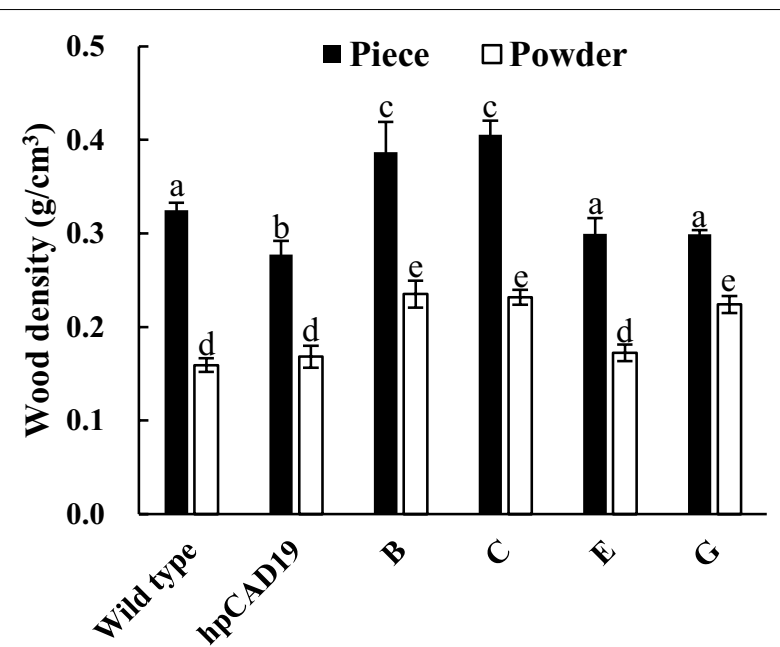

Fig. 5 Densities of dried wood pieces and of cell wall residues (powders) prepared from the stem of each line. Three biological replicates were used in the analysis. Different letters indicate statistically significant differences between the various lines at $P<0.01$ determined by one-way ANOVA followed by Tukey multiple comparison tests. Error bars indicate the standard deviation

Particle density (packed density) was also measured using CWR prepared from stem wood of each line (white bars in Fig. 5). The values were significantly increased in lines $\# \mathrm{~B}$, \#C, and \#G which exhibited higher OsSWN1 overexpression compared to wild type, hpCAD19, and line \#E with lower expression of OsSWN1. Collectively, present results indicate that OsSWN1 overexpression contributes to increase in the stem wood density of the plants with an hpCAD19 background, as reported previously in a wild-type background $[16,25]$.

\section{Lignin characterization}

Lignin content in CWR, as determined by an acetyl bromide protocol, showed no significant differences between the wild-type and the hpCAD19 lines (Additional file 1: Figure S3). Similarly, the lignin contents of lines \#C, \#E, and \#G did not significantly differ from that of their host plant, line hpCAD19. In contrast, that of line \#B was significantly increased by $14 \%$ compared to the hpCAD19 line. This discrepancy observed in the OsSWN1 lines might be due to the expression level of the chimeric transcription factor in each line or the position effect of the gene transfer site in the chromosome.

In addition to the lignin content, the monomeric composition of lignin was also determined by thioacidolysis (Additional file 1: Figure S4). Detected levels of total thioacidolysis monomers ( $G$ plus and $S$ monomers) in the hpCAD19 line were lower than those of wild-type plants, as reported previously [11]. The syringyl to guaiacyl (S/G) ratio of the hpCAD19 line (1.40) was relatively lower than that of the wild-type plants $(1.66, P=0.074)$. Compared to the hpCAD19 plants, the level of the sum of the two monomers was increased in the four OsSWN1 expressing lines but no significant differences were detected. In contrast, the $\mathrm{G}$ monomer level in lines $\# \mathrm{~B}$, \#C, and \#G (which had higher OsSWN1 expression) was significantly higher than that in the hpCAD19 plants. This change led to a significant reduction of the $S / G$ ratio in these three lines $(0.79,0.88$, and 0.98 for the $\# \mathrm{~B}, \# \mathrm{C}$, and \#G lines, respectively) compared to the hpCAD19 line. In line \#E, which displayed relatively low OsSWN1 expression, the S/G ratio (1.29) was comparable to that of the hpCAD19 line. These results suggested that chimeric OsSWN1 expression in plants with an hpCAD19 background were more effective in changing lignin composition than lignin content, as observed in previous studies studying the effects of OsSWN1 expression in a wild-type background [25].

As indicated in Additional file 1: Figure S1, remained CAD1 suppression in the four OsSWN1 lines could be confirmed by real-time PCR. The reduction of CAD1 expression in poplar as well as other plant species known to lead incorporation of coniferaldehyde and sinapaldehyde into the lignin [11]. The incorporation could be detected as release of indene derivatives by thioacidolysis of lignin. Additional file 1: Figure S5 shows the indene release from the four OsSWN1 lines as the hpCAD19 line. In contrast, no release from the wild-type plants could be observed. These results indicate that substantial amount of the hydroxycinnamaldehydes remains to be incorporated into lignin under overexpression of chimeric OsSWN1.

\section{Saccharification efficiency}

Lignin modification via CAD1 suppression improves delignification efficiency under alkaline conditions [15] and the subsequent saccharification of CWRs [11]. Saccharification efficiency was measured after a simple alkaline pretreatment to examine the effect of OsSWN1 expression-induced enhancement of cell wall thickness.

As in the previous study [11], CWR of the hpCAD19 line exhibited high saccharification efficiency compared to wild-type plants (Fig. 6). Although the saccharification efficiency in the OsSWN1-expression lines was still higher than that of the wild-type line, the efficiencies of the $\# \mathrm{~B}, \# \mathrm{C}$, and $\# \mathrm{G}$ lines were slightly but significantly lower than that of the hpCAD19 line. In contrast, no significant difference was observed between the hpCAD19 and line \#E plants. These results indicated that excessive overexpression of chimeric OsSWN1 created a trade-off between enhanced cell wall thickness and improved saccharification efficiency by lignin modification; however, 


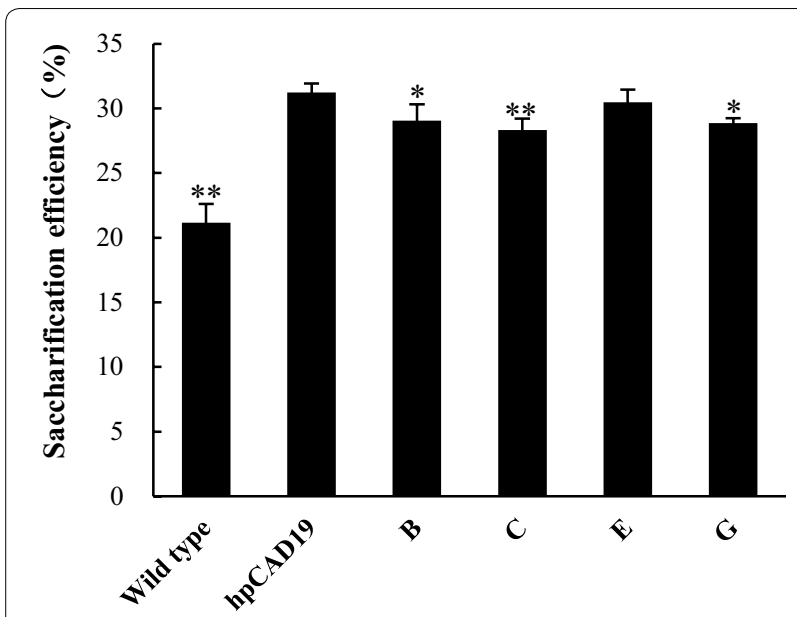

Fig. 6 Enzyme saccharification efficiency of wood powder prepared from the stems of each line. Significant differences from the enzyme saccharification efficiency of the hpCAD19 line at $P<0.05$ and 0.01 are represented as ${ }^{*}$ and ${ }^{* *}$, respectively. A statistical difference was determined by one-way ANOVA followed by Dunnett's test. The values are the mean of three independent biological replicates. Error bars indicate the standard deviation

the improved characteristic of easy delignification by CAD1 suppression remained after cell wall thickness was enhanced by chimeric OsSWN1 overexpression.

\section{Conclusions}

Overexpression of the OsSWN1 transcription factor accelerated SCW deposition in transgenic poplar (hpCAD19), in which the last step of monolignol biosynthesis was suppressed and lignin structure was altered. High overexpression of chimeric OsSWN1 led to an additional modification of lignin structure in the background line and had a slight negative impact on the enzyme saccharification efficiency of the CWR. In order to maximize the synergetic effect of lignin inhibition by $C A D 1$ suppression and enhanced cell wall deposition by OsSWN1 overexpression for efficient lignocellulose production, an in-depth evaluation of the growth retardation mechanism observed in the resultant lines, in addition to the qualitative and quantitative regulation of lignin biosynthesis using the chimeric gene, should be a focus of future studies.

\section{Supplementary information}

Supplementary information accompanies this paper at https://doi. org/10.1186/s10086-020-01902-2.

Additional file 1: Table S1. Nucleotide sequences of primers used in RT-PCR and real-time PCR. Figure S1. CAD1 expression analysis by real-time PCR. CAD1 expression in wild-type plants and transgenic lines were monitored by real-time PCR using CAD1-specific primers. The values were expressed after normalization with the expression of $18 \mathrm{~S}$ ribosomal
RNA gene in each plant. Data were obtained from three biological replicates. Error bars indicate the standard deviation. Different letters indicate statistically significant differences between the wild-type and the transgenic lines at $P<0.01$. A statistical difference was determined by one-way ANOVA followed by Dunnett's test. Figure S2. Moisture content of debarked fresh stems. The value was calculated based on weight of debarked stem wood immediately after harvest compared to air-dried wood. A statistical difference was determined by one-way ANOVA followed by Tukey multiple comparison tests. Significant differences from hpCAD19 line values at $P<0.05$ and 0.01 are represented as * and ${ }^{* *}$, respectively. Error bars indicate the standard deviation. Figure S3. Lignin content in stem wood prepared from different lines. A significant increase in value was only detected in line \#B compared to the wild-type line $(P<0.05)$ by one-way ANOVA followed by Dunnett's test. Error bars indicate the standard deviation. Figure S4. Monomeric composition of lignin evaluated by thioacidolysis and subsequent gas chromatography. Conventional thioacidolysis products with guaiacyl (G) and syringyl (S) nuclei were quantified by gas chromatography equipped with a flameionization detector after trimethylsilylation. Each value is expressed as a relative level of the amount detected in the hpCAD19 line. Significant differences from hpCAD19 line values at $P<0.05$ and 0.01 are represented as * and ${ }^{* *}$, respectively, using one-way ANOVA followed by Dunnett's test. The values are the mean of three independent biological replicates. Error bars indicate the standard deviation. Figure S5. Detection of indene compounds released by thioacidolysis of lignins. Thioacidolysis-released indenes $\left(1_{\mathrm{G} 1}, 1_{\mathrm{S} 1}\right.$, and $\left.1_{\mathrm{S} 2}\right)$, which are indicative of the incorporation of coniferaldehyde and sinapaldehyde into the lignin in CAD suppressed plants, could be detected in the transgenic lines but not in the wild-type plant by GC/MS analysis. Peaks labeled $1_{\mathrm{G} 1}, 1_{\mathrm{S} 1}$, and $1_{\mathrm{S} 2}$ correspond to the indenes with different structures. Detection of the compounds indicates CAD expression is still suppressed in the plants with OsSWN1 as in the hpCAD19 line.

\section{Abbreviations}

CAD: Cinnamyl alcohol dehydrogenase; CWR: Cell wall residue; NAC domain protein: No apical meristem, Arabidopsis transcription activation factor, and cup-shaped cotyledon domain protein; NST3: NAC secondary wall thickening promoting factor 3; OsSWN1: Rice secondary wall NAC domain protein 1; SCW: Secondary cell wall; SND1: SCW-associated NAC domain protein 1.

\section{Acknowledgements}

The authors acknowledge the support from the Institute of Global Innovation Research in Tokyo University of Agriculture and Technology.

\section{Authors' contributions}

$\mathrm{Na}, \mathrm{Nu}, \mathrm{SW}, \mathrm{NT}, \mathrm{SS}, \mathrm{MY}$, and MU performed most of the experiments. NT, AD, $G P, N M$, and SK designed the study and interpreted the data. Na, NT, AD, GP, TT, NM, and SK wrote the manuscript. NT, TT, NM, and SK managed the project. All authors read and approved the final manuscript.

\section{Funding}

This study was supported partially by the ALCA program of the Japan Science and Technology Agency (Grant No. JPMJAL1107).

\section{Availability of data and materials}

The data sets analyzed during the current study are available from the corresponding author upon reasonable request.

\section{Competing interests}

The authors declare that they have no conflict of interest.

\section{Author details}

${ }^{1}$ Graduate School of Bio-Applications and Systems Engineering, Tokyo University of Agriculture and Technology, Koganei, Tokyo 184-8588, Japan. ${ }^{2}$ Forest Bio-Research Center, Forestry and Forest Products Research Institute, Forest Research and Management Organization, Hitachi, Ibaraki 319-1301, Japan. ${ }^{3}$ Bioproduction Research Institute, National Institute of Advanced Industrial Science and Technology (AIST), Tsukuba, Ibaraki 305-8566, Japan. ${ }^{4}$ INRAE, ONF, BioForA, Orléans 45075, France. ${ }^{5}$ Institute of Global Innovation Research, 
Tokyo University of Agriculture and Technology, Fuchu, Tokyo 183-8538, Japan. ${ }^{6}$ Present Address: US Department of Energy's Great Lakes Bioenergy Research Center, Wisconsin Energy Institute, 1552 University Avenue, Madison, WI 53726, USA. ${ }^{7}$ Present Address: Tohoku Regional Breeding Office, Forestry and Forest Products Research Institute, Forest Research and Management Organization, Takizawa, Iwate 020-0173, Japan.

Received: 31 March 2020 Accepted: 22 July 2020

Published online: 06 August 2020

\section{References}

1. Vanholme R, Demedts B, Morreel K, Ralph J, Boerjan W (2010) Lignin biosynthesis and structure. Plant Physiol 153:895-905

2. Boerjan W, Ralph J, Baucher M (2003) Lignin biosynthesis. Annu Rev Plant Biol 54:519-546

3. Boudet AM, Hawkins S, Rochange S (2004) The polymorphism of the genes/enzymes involved in the last two reductive steps of monolignol synthesis: what is the functional significance? C R Biol 327:837-845

4. Ralph J, Hatfield RD, Quideau S, Helm RF, Grabber JH, Jung GH-J (1994) Pathway of $p$-coumaric acid incorporation into maize lignin as revealed by NMR. J Am Chem Soc 116:9448-9456

5. Li M, Pu Y, Ragauskas AJ (2016) Current understanding of the correlation of lignin structure with biomass recalcitrance. Front Chem 4:45

6. Taylor G (2002) Populus: arabidopsis for forestry. Do we need a model tree? Ann Bot 90:681-689

7. Coleman HD, Park JY, Nair R, Chapple C, Mansfield SD (2008) RNAi-mediated suppression of p-coumaroyl-CoA 3'-hydroxylase in hybrid poplar impacts lignin deposition and soluble secondary metabolism. Proc Natl Acad Sci USA 105:4501-4506

8. Van Acker R, Leple JC, Aerts D, Storme V, Goeminne G, Ivens B, Legee F, Lapierre C, Piens K, Van Montagu MC, Santoro N, Foster CE, Ralph J, Soetaert W, Pilate G, Boerjan W (2014) Improved saccharification and ethanol yield from field-grown transgenic poplar deficient in cinnamoyl-CoA reductase. Proc Natl Acad Sci USA 111:845-850

9. Baucher M, Chabbert B, Pilate G, Van Doorsselaere J, Tollier MT, Petit-Conil M, Cornu D, Monties B, Van Montagu M, Inze D, Jouanin L, Boerjan W (1996) Red xylem and higher lignin extractability by down-regulating a cinnamyl alcohol dehydrogenase in poplar. Plant Physiol 112:1479-1490

10. Pilate G, Guiney E, Holt K, Petit-Conil M, Lapierre C, Leple JC, Pollet B, Mila I, Webster EA, Marstorp HG, Hopkins DW, Jouanin L, Boerjan W, Schuch W, Cornu D, Halpin C (2002) Field and pulping performances of transgenic trees with altered lignification. Nat Biotechnol 20:607-612

11. Van Acker R, Dejardin A, Desmet S, Hoengenaert L, Vanholme R, Morreel K, Laurans F, Kim H, Santoro N, Foster C, Goeminne G, Legee F, Lapierre C, Pilate G, Ralph J, Boerjan W (2017) Different routes for conifer- and sinapaldehyde and higher saccharification upon deficiency in the dehydrogenase CAD1. Plant Physiol 175:1018-1039

12. Yamamoto M, Tomiyama T, Koyama A, Okuizumi H, Liu S, Vanholme R, Goeminne G, Hirai Y, Shi H, Takata N, Ikeda T, Uesugi M, Kim H, Sakamoto S, Mitsuda N, Boerjan W, Ralph J, Kajita S (2020) A century-old mystery unveiled: sekizaisou is a natural lignin mutant. Plant Physiol 182:1821-1828

13. Straub CT, Khatibi PA, Wang JP, Conway JM, Williams-Rhaesa AM, Peszlen IM, Chiang VL, Adams MWW, Kelly RM (2019) Quantitative fermentation of unpretreated transgenic poplar by Caldicellulosiruptor bescii. Nat Commun 10:3548

14. Lapierre C, Pilate G, Pollet B, Mila I, Leple JC, Jouanin L, Kim H, Ralph J (2004) Signatures of cinnamyl alcohol dehydrogenase deficiency in poplar lignins. Phytochem 65:313-321
15. Lapierre C, Pollet B, Petit-Conil M, Toval G, Romero J, Pilate G, Leple JC, Boerjan W, Ferret W, De Nadai V, Jouanin L (1999) Structural alterations of lignins in transgenic poplars with depressed cinnamyl alcohol dehydrogenase or caffeic acid O-methyltransferase activity have an opposite impact on the efficiency of industrial kraft pulping. Plant Physiol 119:153-164

16. Sakamoto S, Takata N, Oshima Y, Yoshida K, Taniguchi T, Mitsuda N (2016) Wood reinforcement of poplar by rice NAC transcription factor. Sci Rep 6:19925

17. Zhong R, Lee C, McCarthy RL, Reeves CK, Jones EG, Ye ZH (2011) Transcriptional activation of secondary wall biosynthesis by rice and maize NAC and MYB transcription factors. Plant Cell Physiol 52:1856-1871

18. Mitsuda N, Iwase A, Yamamoto H, Yoshida M, Seki M, Shinozaki K, OhmeTakagi M (2007) NAC transcription factors, NST1 and NST3, are key regulators of the formation of secondary walls in woody tissues of Arabidopsis. Plant Cell 19:270-280

19. Zhong R, Lee C, Zhou J, McCarthy RL, Ye ZH (2008) A battery of transcription factors involved in the regulation of secondary cell wall biosynthesis in Arabidopsis. Plant Cell 20:2763-2782

20. Yoshida K, Sakamoto S, Kawai T, Kobayashi Y, Sato K, Ichinose Y, Yaoi K, Akiyoshi-Endo M, Sato H, Takamizo T, Ohme-Takagi M, Mitsuda N (2013) Engineering the Oryza sativa cell wall with rice NAC transcription factors regulating secondary wall formation. Front Plant Sci 4:383

21. Chai MF, Bellizzi M, Wan CX, Cui ZF, Li YB, Wang GL (2015) The NAC transcription factor OsSWN1 regulates secondary cell wall development in Oryza sativa. J Plant Biol 58:44-51

22. Takata N, Sakamoto S, Mitsuda N, Taniguchi T (2017) The Arabidopsis NST3/SND1 promoter is active in secondary woody tissue in poplar. J Wood Sci 63:396-400

23. Leple JC, Brasileiro AC, Michel MF, Delmotte F, Jouanin L (1992) Transgenic poplars: expression of chimeric genes using four different constructs. Plant Cell Rep 11:137-141

24. Takata N, Taniguchi T (2015) Expression divergence of cellulose synthase (CesA) genes after a recent whole genome duplication event in Populus. Planta 241:29-42

25. Nuoendagula, Tsuji Y, Takata N, Sakamoto S, Nakagawa-Izumi A, Taniguchi T, Ralph J, Mitsuda N, Kajita S (2018) Change in lignin structure, but not in lignin content, in transgenic poplar overexpressing the rice master regulator of secondary cell wall biosynthesis. Physiol Plant 163:170-182

26. Dougherty R, Kunzelmann K (2007) Computing Local Thickness of 3D Structures with ImageJ. Microsc Microanal 13:1678-1679

27. Yue F, Lu F, Sun RC, Ralph J (2012) Syntheses of lignin-derived thioacidolysis monomers and their uses as quantitation standards. J Agric Food Chem 60:922-928

28. Miller GL (1959) Use of dinitrosalicylic acid reagent for determination of reducing sugar. Anal Chem 31:426-428

29. Fukushima K, Imai T, Terashima N (1994) Heterohenous lignification in one-year-old shoots of trees I. Characterization of cell wall components in the various tissues of a one-year-old poplar shoot. Mokuzai Gakkaishi 40:958-965

30. Özparpucu M, Gierlinger N, Burgert I, Van Acker R, Vanholme R, Boerjan W, Pilate G, Dejardin A, Ruggeberg M (2018) The effect of altered lignin composition on mechanical properties of cinnamyl alcohol dehydrogenase (CAD) deficient poplars. Planta 247:887-897

\section{Publisher's Note}

Springer Nature remains neutral with regard to jurisdictional claims in published maps and institutional affiliations. 